Europhys. Lett., 63 (6), pp. 902-907 (2003)

\title{
Effect of surfactant structure on interfacial properties
}

\author{
L. Rekvig $\left.{ }^{1}{ }^{*}\right)$, M. Kranenburg ${ }^{2}$, B. Hafskjold ${ }^{1}$ and B. Smit $^{2}$ \\ 1 Department of Chemistry, Norwegian University of Science and Technology \\ N-7491 Trondheim, Norway \\ 2 Department of Chemical Engineering, University of Amsterdam \\ Nieuwe Achtergracht 166, 1018 WV Amsterdam, The Netherlands
}

(received 15 April 2003; accepted in final form 11 July 2003)

PACS. 82.70.Uv - Surfactants, micellar solutions, vesicles, lamellae, amphiphilic systems (hydrophilic and hydrophobic interactions).

PACS. 68.05.-n - Liquid-liquid interfaces.

\begin{abstract}
We study surfactants at the oil/water interface using Dissipative Particle Dynamics simulations at constant $\mu_{\text {surf }} P T$. The interfacial tension depends on the surfactant branching in a subtle way. For a given interfacial concentration, a double-tail surfactant is more efficient than its single-tail isomer only if the oil-head repulsion is sufficiently strong. For a given concentration in the bulk water phase, the single-tail surfactants are more efficient in both cases. We interpret these results in light of the molecular packing at the interface and free-energy considerations.
\end{abstract}

Introduction. - Surfactants are molecules that consist of hydrophobic and hydrophilic parts. Their amphiphilic nature makes them surface active and, adsorbed at the oil/water interface, they can reduce the bare oil-water interfacial tension to very low values. Because of this property, surfactants are used in many practical applications ranging from crude oil recovery to state-of-the-art drug delivery [1] and are also of scientific interest. From a practical viewpoint, it is important to understand how the efficiency in reducing the interfacial tension is related to the structure of a surfactant. This question was already posed by Traube in 1899 [2] and he discovered that increasing the hydrophobic tail length results in surfactants that are more efficient (Traube's rule). At present, the effect of branching of the hydrophobic tail is not yet fully understood, despite the fact that many of the surfactants used in industrial applications are prepared with branched hydrocarbon tails. The effect of branching on the interfacial tension was investigated by self-consistent field calculations [3] and molecular-dynamics simulations on model surfactants [3,4]. These studies agree on the fact that surfactants with two hydrophobic chains are less efficient in reducing the interfacial tension compared to their single-tail isomers. Experimentally, however, either more, equal, or less efficient branched surfactants are reported, depending on the details of the experimental setup [3,5-8].

$\left(^{*}\right)$ On leave at Department of Chemical Engineering, University of Amsterdam, Amsterdam, The Netherlands. E-mail: live.rekvig@phys.chem.ntnu.no

(C) EDP Sciences 


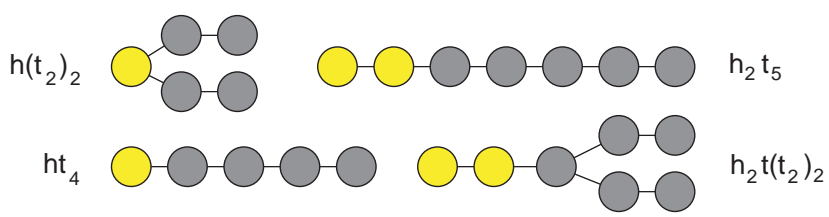

Fig. 1 - Some of the model surfactants and their respective names. Hydrophilic head beads (h) are shown in yellow and hydrophobic tail beads (t) are shown in gray.

Although it is assumed that branched molecules pack differently at the interface compared to linear ones $[9,10]$, a molecular description of their tension-reducing properties is, to the best of our knowledge, still missing. In this work, we use Dissipative Particle Dynamics [11] (DPD) simulations to study the effect of branching on the interfacial properties of surfactants. Our simulations demonstrate that the head group properties determine the effect of branching on the interfacial tension, and we explain this by means of packing and ordering of the molecules at the interface. Furthermore, the efficiency also depends on the partitioning of the surfactant between the bulk liquid and the interface. Molecular simulation of a system with an interface in contact with its bulk phases cannot be used to effectively determine the bulk surfactant concentration due to the very low concentrations and long diffusion times involved. Here we present a method to overcome this problem, and report simulation results of interfacial tension $v s$. surfactant concentration in the bulk for both single-tail and double-tail surfactant structures.

Method and model. - In a DPD simulation one uses dissipative and random forces in addition to the conservative forces between the particles. The dissipative and random forces are chosen such that a proper Boltzmann distribution of configurations is sampled corresponding to the intermolecular interactions from which the conservative forces are derived [12]. In analogy with previous simulations of surfactants using the DPD technique [13], we use softrepulsive interactions to mimic the mesoscopic interactions between the oil, water, and surfactant molecules. In our model, we distinguish four types of particles, o, w, h, and t, to mimic the oil, water, and the head and tail molecular groups of a surfactant, respectively. For the conservative forces we use the conventional soft repulsive interactions $F(r)=a\left(1-r / r_{\text {cut }}\right)$ in which the parameters $a$ are chosen such as to mimic the hydrophobic and hydrophilic interactions: $a_{\mathrm{ww}}=a_{\mathrm{oo}}=25, a_{\mathrm{hh}}=35, a_{\mathrm{wo}}=a_{\mathrm{oh}}=80, a_{\mathrm{wh}}=15$. The tail particles are identical to the oil particles. Our parameters are similar to those optimized by Groot [14], and reproduce the compressibility of water and solubility of non-ionic surfactant segments. The surfactant particles are connected via harmonic springs, with spring constant $k=100$ and equilibrium distance $r_{0}=0.7$. Some of the surfactants models are pictured in fig. 1 . This model maps typically 3-6 $\mathrm{CH}_{2}$ groups onto one tail bead [13-15]. We simulated a system with approximately 8000 particles at temperature $T=1.0$ and pressure $P=23.6$ corresponding to a bulk density of 3.0. The box area was $11 \times 11$ and doubling the system size did not alter the results. All properties are expressed in the usual reduced units, i.e. using $r_{\text {cut }}$, repulsion parameter $a=1$, and the mass of a DPD bead as units of length, energy, and mass, respectively. The interfacial tension was calculated by integrating the difference in normal and tangential pressure [16].

In previous simulations the interfacial tension was studied as a function of the total number of surfactants added to the system $[3,4,16,17]$, whereas experimentally one usually determines the concentration of surfactants in the bulk phase. To determine the interfacial tension in a simulation one simulates the oil/water interface explicitly. In such an inhomogeneous system it is difficult to determine the concentration of the surfactants accurately. One not only needs relatively large systems to minimize the influence of the interface, but also very long 


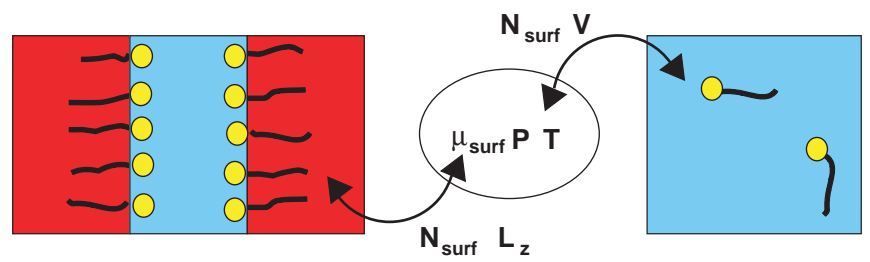

Fig. 2 - Simulation setup; the left figure is the inhomogeneous system that contains an oil/water interface and surfactants and the right figure is the homogeneous system containing water and surfactants. Both systems are coupled to a reservoir, which imposes the temperature, (normal) pressure, and chemical potential of the surfactants.

simulations since the concentration of surfactants in the bulk phase is usually very low. To determine the interfacial tension as a function of the surfactant concentration in the bulk, we introduce an ensemble in which we impose the temperature, (normal) pressure, and the chemical potential of the surfactant $\left(\mu_{\text {surf }}\right)$, see fig. 2 . In this ensemble two systems are simulated; an inhomogeneous system containing the oil/water interface and a homogeneous system that contains the bulk water phase. The conventional DPD simulations, performed at constant number of particles $(N)$, volume $(V)$, and temperature, are combined with Monte Carlo moves in which we attempt to change the number of surfactant particles and a move in which we change the volume. For the inhomogeneous system we change the volume in such a way that the area of the oil/water interface remains constant, i.e. imposing the normal pressure. For a pure component one cannot impose both the chemical potential and the pressure, the extensive variables $N$ and $V$ would be unbounded [12]. In our system we keep the number of water (and oil) particles constant, hence fixing at least one extensive variable.

Similar to grand-canonical ensemble simulations, our ensemble relies on the successful insertion/deletion of the particles in the system. For atoms or small molecules this can be achieved by random insertion of the particles. For chain molecules, however, random insertion is very inefficient. To make this type of insertion moves possible for the surfactant molecules, we use the configurational-bias Monte Carlo technique [18] (CBMC). In a CBMC simulation a molecule is grown atom by atom in such a way that the "empty" spaces in the system are found. The bias introduced by this growing algorithm is removed exactly by adjusting the acceptance rules. With this CBMC scheme we obtain a sufficient number of accepted insertion and deletions in the inhomogeneous system to determine the interfacial concentration and tension. For our surfactant models, the concentrations in bulk water were found to be sufficiently low for Henry's law to be valid:

$$
\frac{N_{\text {surf }}}{V}=K_{\mathrm{H}} P_{\text {surf }}
$$

Here $K_{\mathrm{H}}$ is the Henry coefficient and $P_{\text {surf }}$ is the effective partial pressure of the surfactants in the reservoir. $P_{\text {surf }}$ is directly related to $\mu_{\text {surf }}$, which is imposed in the inhomogeneous system. $K_{\mathrm{H}}$ is related to the excess chemical potential at infinite dilution:

$$
K_{\mathrm{H}}=\beta \exp \left[-\beta \mu_{\mathrm{surf}}^{\text {excess }}\right] \text {. }
$$

$\mu_{\text {surf }}^{\text {excess }}$ can be computed using a test particle method based on the CBMC scheme [12].

Interfacial tension vs. interfacial density. - Figure 3a) shows the interfacial tension as a function of surfactant concentration at the interface for various surfactant structures. At 

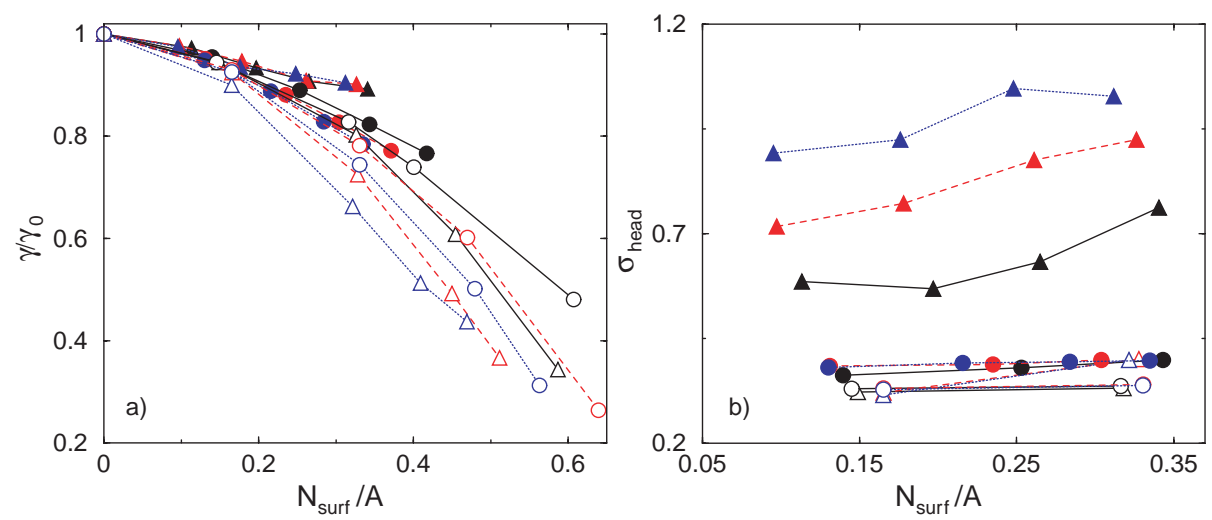

Fig. 3 - a) Reduced interfacial tension $\left(\gamma / \gamma_{0}\right)$ as a function of the surfactant concentration at the interface $\left(N_{\text {surf }} / A\right)$ for some single-tail $\left(\mathrm{ht}_{4}, \mathrm{ht}_{6}, \mathrm{ht}_{8}\right)$ and double-tail $\left(\mathrm{h}\left(\mathrm{t}_{2}\right)_{2}, \mathrm{~h}\left(\mathrm{t}_{3}\right)_{2}, \mathrm{~h}\left(\mathrm{t}_{4}\right)_{2}\right)$ surfactants with high and low oil-head repulsion $a_{\mathrm{oh}}$. The circles are for the single-tail surfactants and the triangles for the double-tail isomers, the open symbols indicate $a_{\mathrm{oh}}=80$ and the filled symbols $a_{\mathrm{oh}}=30$. The surfactants have 4 (black, solid lines), 6 (red, dashed lines), and 8 (blue, dotted lines) tail beads. b) Ordering at the interface as a function of the concentration of molecules at the interface. The ordering is quantified as the width of the distribution, $\sigma_{\text {head }}$, of surfactant head groups at the interface.

low concentration we see that the different surfactant structures cause a similar reduction of the interfacial tension. At these low interfacial concentrations the surfactants do not interact and form a two-dimensional ideal gas at the interface. At higher concentrations the surfactants interact and we observe differences between the various structures. For single-tail surfactants (open circles), increasing the tail length results in more efficient surfactants, because of the increased excluded-volume interactions between the tails [19]. Our simulations show that double-tail surfactants, depending on the oil-head interactions, can be either more or less efficient compared to their single-tail isomers. For high values of the oil-head repulsion parameter $a_{\mathrm{oh}}=80$ we find the double-tail isomers to be more efficient, while for $a_{\mathrm{oh}}=30$ we find the single-tail isomers to be more efficient.

To obtain a molecular understanding of the results we investigate the ordering and packing of the surfactants at the interface. A schematic picture of these results is shown in fig. 4 . We have computed the width of the distribution of the head groups normal to the interface. Figure $3 \mathrm{~b}$ ) shows that for both the single-tail and double-tail surfactants decreasing the oilhead repulsion results in a broader distribution but this effect is much more pronounced for double-tail molecules. We also investigated the degree of alignment of the bond between hydrophilic and hydrophobic beads with the interfacial normal. The double-tail surfactants lose more bond order than the single-tail isomers when the repulsion parameter is lowered (not
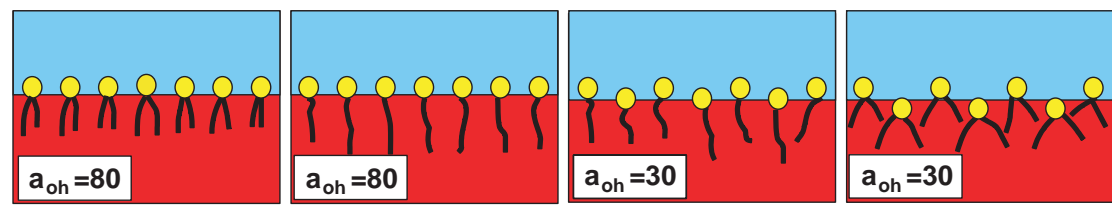

Fig. 4 - Schematic picture of singe-tail and double-tail surfactants at the oil/water interface. The four surfactant types are arranged from left to right in order of decreasing efficiency. 


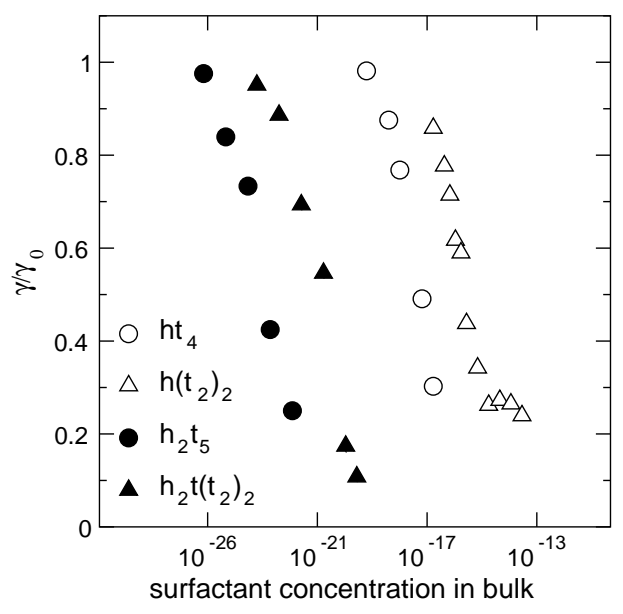

Fig. 5 - Reduced interfacial tension $\left(\gamma / \gamma_{0}\right)$ as a function of the bulk surfactant concentration $\left(N_{\text {surf }} / V\right)$ in the water phase for $a_{\mathrm{oh}}=80$.

shown). Inspection of the snapshots of the simulation shows that the molecular explanation of the broadening and the loss of bond order is that the molecules with less hydrophobic headgroups stagger at the interface, as shown schematically in fig. 4 . In such an arrangement the excluded-volume interactions are far less efficient in reducing the interfacial tension.

If the oil-head interaction is sufficiently repulsive, the surfactants order nicely at the interface. The distribution of the head groups normal to the interface is narrow and the doubletail molecules experience stronger excluded-volume interactions than the single-tail ones. If the oil-head interaction is decreased, the double-tail molecules stagger, resulting in weaker excluded-volume interactions between them compared to the single-tail isomers. Hence, if we use surfactants with sufficiently hydrophilic head groups, double-tail surfactants are more efficient than single-tail ones for a given interfacial concentration. It is interesting to compare our results with the theoretical calculations $[3,19]$. In these theories the head groups are assumed to be in a fixed plane (i.e. fig. 4, left). With this assumption, the surfactants are not allowed to adopt a staggered conformation (i.e. fig. 4, right).

Interfacial tension vs. bulk concentration. - When we compare the interfacial tension as a function of the bulk concentration of surfactants the picture changes. Figure 5 shows that although the double-tail isomers are more efficient for a given interfacial concentration, they are less efficient for a given bulk concentration.

The reason for this is as follows: At a given interfacial concentration the effective repulsion between the tails is higher compared to their single-tail isomers (see fig. 4). As a consequence, the chemical potential of these double-tail isomers is significantly higher for the same interfacial concentration. However, in bulk water the Henry coefficient is of the same order of magnitude for single-tail and double-tail molecules. This implies that the concentration of double-tail surfactants in the bulk water phase must be much higher to yield the same (equilibrium) concentration at the interface. At this point, it is important to note that our results on the efficiency should not be confused with the effectiveness of a surfactant, defined as the lowest interfacial tension that can be reached by adding surfactant. The effectiveness is usually determined by the interfacial tension at the critical micelle concentration (CMC). If the CMC for a double-tail surfactant is much higher compared to its single-tail isomer, lower interfacial tension can be obtained by using double-tail surfactants. 
For the models we have studied, the efficiency at the interface of the double-tail surfactants did not compensate for the reduced ease of adsorption. It is therefore tempting to conclude that it is not possible to synthesize double-tail surfactants that require a lower bulk concentration to reduce the interfacial tension by the same amount as their single-tail isomers. Of course, in our model we have coarse-grained all interactions into very simple potentials. In this process we may have lost the subtleties that may make real double-tail surfactants more efficient than their single-tail isomers.

Summary. - We have shown that a bulk solution in equilibrium with an interface can be simulated using a constant $\mu_{\text {surf }} P T$ ensemble. Moreover, the ordering of head groups normally to the interface can explain why branching of the hydrophobic tail can have either a positive or a negative effect on a surfactant's tension-reducing abilities. Finally, for doubletail surfactants there are two opposing effects: the more efficient the surfactants are at the interface, the higher the bulk concentrations required to get them to the interface.

$$
* * *
$$

These investigations are supported in part by the Norwegian Research Council (grant no. 145184/432), by the Netherlands Research Council for Chemical Sciences (CW), and by the Netherlands Organization for Scientific Research (NWO) through PIONER.

\section{REFERENCES}

[1] Tsuji K., Surface Activity: Principles, Phenomena, and Applications (Academic Press, San Diego) 1998.

[2] Traube J., Samml. Chem. Vortr., 4 (1899) 255.

[3] van Os N. M., Rupert L. A. M., Smit B., Hilbers P. A. J., Esselink K., Böhmer M. R. and Koopal L. K., Colloids Surf. A, 81 (1993) 217.

[4] Klopfer K. J. and Vanderlick T. K., Colloids Surf. A, 96 (1995) 171.

[5] Varadaraj R., Bock J., Valint P. JR., Zushma S. and Thomas R., J. Phys. Chem., 95 (1991) 1671.

[6] Pitt A. R., Morley S. D., Burbidge N. J. and Quickenden E. L., Colloids Surf. A, 114 (1996) 321.

[7] Wormuth K. R. and Zushma S., Langmuir, 7 (1991) 2048.

[8] Aspée A. and Lissi E, J. Colloid Interface Sci., 178 (1996) 298.

[9] Ghaїcha L., Leblanc R. M. and Chattopadhyay A. K., J. Phys. Chem., 96 (1992) 10948.

[10] Green S. R., Su T. J., Lu J. R. and Penfold J., J. Phys. Chem. B, 104 (2000) 1507.

[11] Hoogerbrugge P. J. and Koelman J. M. V. A., Europhys. Lett., 19 (1992) 155.

[12] Frenkel D. and Smit B., Understanding Molecular Simulations: from Algorithms to Applications, 2nd edition (Academic Press, San Diego) 2002.

[13] Prinsen P., Warren P. B. and Michels M. A. J., Phys. Rev. Lett., 89 (2002) 148302.

[14] Groot R. D., Langmuir, 16 (2000) 7493. To obtain similar bulk densities for the water and oil phase we used $a_{\mathrm{oo}}=25$ instead of 15 used by Groot.

[15] Groot R. D. and Rabone K. L., Biophys. J., 81 (2001) 725.

[16] Smit B., Phys. Rev. A, 37 (1988) 3431.

[17] Smit B., Schlijper A. G., Rupert L. A. M. and van Os N. M., J. Phys. Chem., 94 (1990) 6933.

[18] Frenkel D., Mooij G. C. A. M. and Smit B., J. Phys. Condens. Matter, 4 (1992) 3053.

[19] Szleifer I., Ben-Shaul A. and Gelbart W. M., J. Phys. Chem., 94 (1990) 5081. 